\title{
Two class medicine: the essence hits it all
}

\author{
F. M. Riegler
}

Published online: 19 August 2014

(C) Springer-Verlag Wien 2014

\section{Dear readers,}

Based on the Kantian approach towards the separation of perceptions into classes and categories, modern life sorts out two classes of medicine-the first and the second class medicine. The present time operates a largely greed- and economy-driven way of thinking. Therefore, at first sight, the two quality classes of medicine are interpreted as low and high cash volume medicine. Thus, in the eyes of the physician, social security system covered health service is considered the low cash volume medicine. As a matter of fact it seems to be overseen that what the servant and the serviced do not see in fact still exists, i.e., the economic burden. And thus it is, because the services are to be outbalanced by the community health system. However, the salary of the physicians working in the community health service is somehow smaller, lower, when compared with the cash possibilities in the so called "first"class private medicine market. In contrast, the so called cash advantages and opportunities of the private medicine market are outbalanced by the increased risks of the private economy shareholders. Thus, the holistic view seems to justify a similar end of the day with both ways (Fig. 1). Yet, it remains to be questioned if there may exist a quality based classification of medicine?

Using an essence based approach, there exists a good and a bad medicine. Good medicine assesses and treats the essence of a disease; this is the basic ground of the impairment of life quality and productivity (WHO are YOU?), and leads to well being and health. Conceptually all conditions covered within a text book of internal medicine are abnormal reactions to the consumption of concentrated carbohydrates $(=$ the centered nucleus of

F. M. Riegler ( $\square)$

Reflux Medical,

Mariannengasse 10/9,

1090 Vienna, Austria

e-mail: martin.riegler@refluxmedical.com the metabolism). Exceptions include obstetrics, trauma, and malformations.

And thus goes the late Johnny Winter reggae blues: at some point in life the digestion of concentrated carbohydrates commences to steal energy from the organism, instead of providing energy. As a consequence the organism lacks energy and this in turn activates the nerve cells to provoke a nerve cell mediated response. Due to the lack of energy the nerve cells release a cocktail of beautiful hormone proteins into the tissues (substance $\mathrm{P}$, neurotensin, adrenalin, serotonin, vasoactive intestinal peptide etc.). This cocktail elicits inflammation, pain, and dysfunction. Gut cries the tears of cold sweet sweat diarrhea. Depending on the individual hereditary genetic profile this cocktail of proteins starts to affect the function and being of the genetically "weak" organs. For example, as a response to the nerve-cell released proteins the vascular system and lung system contracts to generate hypertension and asthma, respectively. In the gut the release of the cocktail provokes heartburn, gas bloat, irritable bowel syndrome, inflammatory reactions (Crohn's disease, ulcerative colitis) and diabetes (if the imbalance hits the pancreas!). If the cocktail affects the skin, patients develop neurodermatitis, skleroderma or the lupus concert of tragedy. Nerve cells within vessels and heart provoke the development of arteriosklerosis and angina pectoris. Furthermore, the cocktail may be released into the brain. As a consequence patients are tired after a meal or they develop depression. Finally, if the cocktail outbalances the genetics polyps, tumors and cancers may grow out within the darkness of the hidden shine. Taken together, the food induced energy lack drives a nerve cell mediated imbalance of the metabolism. Unfortunately, most drugs contain concentrated sugar compounds, thus provoking the disease that they are intended to treat. It remains to be questioned how this concept may translate into a better, a good class medicine? 
Fig. 1 Every day times out with the sunset, irrespective of the way the cosmos scattered the space. The image mirrors the idea of the author regarding the misconception of first and second class medicine. Image obtained using iPhone technology in Sicily, EU

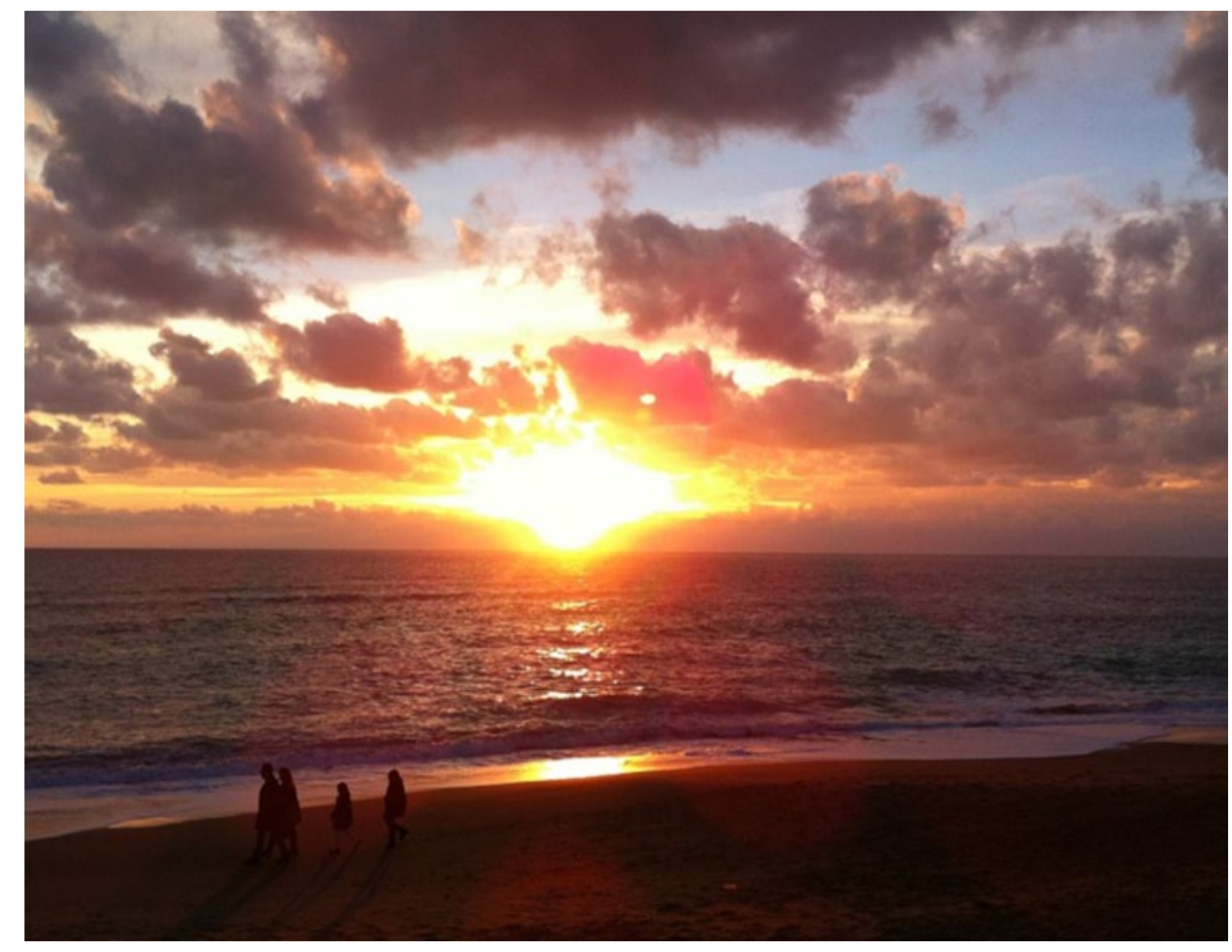

Evidence indicates that diseases and their reported co-morbidities share a common origin, i.e., the nutrition induced imbalance of the metabolism, i.e., obesity, diabetes, hypertension, asthma, stroke, depression, neurodermatitis, gastroesophageal reflux disease, allergies, and cancer turn out to exist in parallel. Furthermore, elimination of concentrated sugars from the diet fosters to improve the so called dominant disease and the so called co-morbidities. Otherwise the imbalance may have developed due to an unknown, not yet discovered energy-eater, i.e., tumor, polyp, inflammation. Following elimination of such an energy eater and outbalanced carbohydrate-diet concept should work out to orchestrate health and well being. In addition, interpretation of clinical trials should try to include the above concept of reasoning. Then we may better understand why premalignant conditions of the gut (+ respective therapies) are paralleled by an increased risk for cardiac dysfunction or stroke, pneumo- nia, or migraine. It seems time is ready to question the current concepts, otherwise we will increasingly observe that the metabolic imbalance will strike out the Western civilization and those coming from the continent, where we all came from, will come to take (us) over: the Africans. And at the very end it seems we will have to go there, where all life comes from: back to the sea, oceans, and the universe. A star gives the light to the rainbow. Cosmos is the tension within the beam. Like water drops, life will come and go along the spirit of the universal wheel: lack fosters being. Diseases are the different masks of the essence, the metabolic imbalance; it is all the same, see how easy medicine may be, if the idea opens for the common ground. Enjoy life and the spring falls of autumn.

\section{Conflict of interest}

The author declares that there exists no conflict of interest. 